\title{
Symposium «ZNS-Metastasen des Mammakarzinoms» am 21. Oktober 1989 in Berlin
}

Der interdisziplinären Thematik entsprechend wurde dieses Symposium von den

Arbeitsgemeinschaften Neuroonkologie, Internistische Onkologie, Radiologische Onkologie und Gynäkologische Onkologie der Deutschen Krebsgesellschaft getragen. Themen waren die Hirnmetastasen, die Meningeosis carcinomatosa und die Rückenmarkkompression. ZNSMetastasen sind beim Mammakarzinom ein Ereignis, das eher im fortgeschrittenen Stadium der Erkrankung auftritt (Wander, Göttingen). Es ist wahrscheinlich, daß Hirnmetastasen und die Meningeosis carcinomatosa besonders bei auch im übrigen aggressiven Verläufen der Erkrankung wie z.B. bei Hormonrezeptor-negativen Tumoren und im Zusammenhang mit anderen parenchymatösen Metastasen von Bedeutung sind. Das klinische Bild kann sehr uncharakteristisch sein und erfordert daher vom betreuenden Arzt eine besondere Aufmerksamkeit (Krauseneck, Würzburg).

Die Computertomographie ist für die Diagnose der Hirnmetastasen wegen ihrer größeren Verbreitung die Methode der Wahl (Steinbrich, Köln). Nur bei unklaren Befunden, insbesondere an der Schädelbasis und in der hinteren Schädelgrube, sollte die an sich sensitivere Kernspintomographie Verwen-dung finden (Schörner, Berlin). Bei klinischem Verdacht auf Rückenmarkkompression führt häufig die Kombination von Myelographie und Computertomographie zur Diagnose (Thron, Aachen). Aber auch in diesem Bereich ist die Kernspintomographie empfindlicher, so daß bei eindeutiger Klinik und negativer Computertomographie hiermit die Diagnose rasch mit hoher Sicherheit gestellt werden kann. Für die Diagnose der Meningeosis carcinomatosa gibt es kein zuver-lässiges bildgebendes Verfahren (Kölmel, Berlin). Der Nach-weis von Tumorzellen im Liquorpunktat, notfalls auch nach mehrmaliger Punktion, ist für die Stellung der Diagnose erfor-derlich.

Die Operationsindikation für Hirnmetastasen des Mammakar-zinoms wurde eindeutig bejaht unter der Voraussetzung, daß es sich um eine solitäre Metastase handelt und daß keine ausgedehnte systemische Manifestation der Erkrankung vor-liegt (Grote, Tubingen). Die Frage, ob im Anschluß an eine solche Operation eine adjuvante Strahlentherapie des Gehirns erfolgen solle, wurde kontrovers diskutiert. Dagegen gilt die Strahlentherapie des Gehirns bei multipler oder diffuser Meta-stasierung als etabliertes Behandlungsverfahren (Bamberg, Tubingen). Dabei ist eine Dosis von 30 Gy über einen Zeit-raum von zwei Wochen auf das ganze Hirn appliziert wahrscheinlich ausreichend. Sehr hohe Dosen führen zu unzumut-baren Nebenwirkungen (Sartorelli, Zurich). In Konkurrenz zur Strahlentherapie steht die systemische medikamentöse Behandlung mit Hormonen und/oder Zytostatika (Kreuser, Berlin). Grundsätzlich können Hirnmetastasen auch auf eine systemische Therapie ansprechen. Allerdings liegen keine ausreichenden systematischen Untersuchungen vor, die die Art der Vorbehandlung berücksichtigen. Patientinnen, die bereits mehrfach hormonell und/oder zytostatisch vorbehandelt sind, werden wahrscheinlich von einer erneuten systemischen Therapie weniger profitieren als von einer Bestrahlung. Die Therapie der Meningeosis carcinomatosa des Mammakarzmoms besteht 
in der intrathekalen Applikation von Metho-trexat (Herrmann, Berlin). Kombinationen mit anderen Zytostatika wie z.B. Cytosinarabinosid und ThioTEPA oder mit gleichzeitiger Bestrahlung des Liquorraums sind nicht besser. Eine Indikation zum orthopädisch-chirurgischen Eingreifen ergibt sich bei Rückenmarkkompression im Zusammenhang mit einer Wirbelsäulenmetastasierung (Weber, Berlin). Prinzi-pien des Vorgehens sind der Zugang am Erkrankungsort, also überwiegend von ventral, und die Wiederherstellung der Sta-bilität. Übereinstimmung bestand darin, daß die Laminekto-mie als dorsales Entlastungsverfahren bei ventraler Markkom-pression ein ungeeignetes Verfahren darstellt. Das Rücken-mark komprimierende Metastasen, die nicht von der Wirbel-säule ausgehen, werden neurochirurgisch behandelt (Schön-mayr, Gießen). Allerdings ist bei Laminektomien über mehr als eine Etage ein gemeinsames Vorgehen mit dem Orthopä-den anzustreben, um die Stabilität der Wirbelsäule zu sichern. Die Strahlentherapie der Rückenmarkkompression stellt einerseits eine Alternative, andererseits eine Ergänzung des operativen Vorgehens dar (Wannenmacher, Heidelberg). Bei Patientinnen, die die Operation ablehnen, die aus anderen Gründen nicht operabel sind oder deren Prognose aufgrund einer weit fortgeschrittenen Erkrankung sehr limitiert ist, stellt die Bestrahlung eine wirksame Alternative dar. Ferner sollte in der Regel nach operativer Ausräumung und Stabili-sierung eine Nachbestrahlung des betroffenen Gebietes erfolgen, da eine lokale Sanierung durch Operation allein praktisch nicht möglich ist.Die ZNSMetastasierung des Mammakar-zinoms stellt für die Patientinnen eine besondere Bedrohung dar. Für den behandelnden Arzt ist sie eine große Herausfor-derung. Wie kaum eine andere Metastasenlokalisation erfordert sie für Diagnostik und Therapie eine enge effektive interdisziplinäre Absprache. Dazu gehört auch die Einord-nung der ZNS-Metastasierung in den individuellen Gesamt-verlauf einer Tumorerkrankung, welche eine Abschätzung der Prognose und damit eine sinnvolle individuelle Therapie-planung ermöglicht.

R. Herrmann, Berlin 